\title{
A Non-perturbative Model of Mean Field Valence Quarks
}

\section{Christopher Leon*}

Department of Physics, Florida International University, Miami, Florida 33199, USA

E-mails: cleon082@fiu.edu

\section{Misak Sargsian}

Department of Physics, Florida International University, Miami, Florida 33199, USA

E-mail: sargsianefiu.edu

\begin{abstract}
We present a new approach for the calculation of the valence quark distributions in the nucleon based on the scenario in which the spectrum of the valence quarks at $x>0.05$ is generated through three main mechanisms: interaction of valence quarks with the mean field generated by the residual nucleon system, two and three quark short range interactions through gluon exchanges. In the current report we present the first phase of the project in which we develop a non-perturbative model for valence quark interaction in the mean field of the nucleonic interior to describe their distribution in the moderate $\mathrm{x}$ region $(0.05 \leq x \leq 0.4)$. The short range quark-quark interaction effects in our approach generate the high $x$ tail of valence quark distributions. The presented nonperturbative model is based on the picture in which three relativistic valence quarks occupy the nucleon core at distances of $\leq 0.5 \mathrm{Fm}$ while interacting in the mean field generated by the residual nucleon system. The calculations are based on the assumption of the a factorization of the internal interaction of short-range three valence quarks with the long-range interaction of these quarks with the residual system. The theoretical approach is based on effective light-front diagrammatic approach which allows us to introduce the valence quark and residual system wave functions in a consistent way The parameters of these wave functions are fixed by the position of the peak of the $x f_{q}(x)$ distribution of valence quarks at $Q_{0}$ corresponding to the charm-quark mass. With few parameters we achieved a very reasonable description of the up and down valence quark distributions in the moderate $x$ region $(x \leq 0.4)$, where one expects the mean field dynamics to dominate. The model, however, systematically underestimates the high $x$ region where enhanced contributions from partonic short-range correlations are expected.
\end{abstract}

Light Cone 2019 - QCD on the light cone: from hadrons to heavy ions - LC2019

16-20 September 2019

Ecole Polytechnique, Palaiseau, France

${ }^{*}$ Speaker. 


\section{Introduction}

Understanding the dynamics of the valence quarks in the nucleon is one of the most interesting issues of quantum chromodynamics (QCD). The special aspect of valence quarks is that they define the spin and isospin quantum numbers of the nucleon and one expects them to be a bridge between baryonic spectroscopy and the QCD structure of the nucleon.

The history of the modeling partonic distributions is very rich ranging from nonrelativistic and relativistic constituent quark models, bag models, models combining the partonic and pionic cloud pictures of the nucleon, as well as models based on the di-quark picture of the nucleon (see Ref.[1,2] and references therein). All these models are non-perturtbative in their nature and their predictions vary widely for the general characteristics of the valence quark distribution, such as position of the peak and relative strength of the x-weighted $u$ - and $d$-quark distributions. While calculations based on lattice QCD reproduce the general characteristics of the valence quark distribution, their complexity does not always allow an understanding of the dominant mechanisms in the generation of valence quark distributions.

In this report we present a development of a new model for valence quark distributions of the nucleon, based on the multi-quark correlation picture. The validity of such model is based on the fact that even though the number of the quarks are not conserved in the nucleon the number of valence quarks are "effectively" conserved and therefore it is possible to describe them in the framework used for the description of a bound system of a finite number of fermions. This approach is similar to the highly successful multi-nucleon correlation model of calculation of momentum distributions of nucleon in the nuclei (see e.g. [3]).

In this approach we consider three distinct interaction dynamics of valence quarks which are: mean field, two-quark and three-quark short range correlations, all of which dominate at different momentum fraction ranges of valence quarks. The advantage of the proposed framework is that it creates a new ground for making predictions for different QCD processes involving nucleons, since in this case one can make unique predictions based on whether the process under the study is dominated by the interaction of quarks in the mean field or two-/three- quark correlations.

\section{Phenomenology of valence PDFs and basic assumption of the model}

Since baryonic spectroscopy adheres reasonably well to SU(6) spin-flavor symmetry, one expects such a symmetry to be found also in the valence quark distributions in the nucleon. For valence quark distributions the $\mathrm{SU}(6)$ symmetry will result in the ratio of $d_{V}$ to $u_{V}$ quark distribution to be: $\frac{d_{V}\left(x, Q^{2}\right)}{u_{V}\left(x, Q^{2}\right)}=\frac{1}{2}$.

As it can be seen in Fig. 1 the experimental extractions of the valence quark distributions indicate that the $\mathrm{SU}(6)$ condition is satisfied to some extent for the region of $0.1<x<0.3$ and then it is progressively violated with the increase of $x$.

One intriguing feature following from Fig.1 (top and bottom panels) is that the approximate agreement with $\mathrm{SU}(6)$ symmetry takes place in the domain of Bjorken $x$ where valence quark distributions weighted by Bjorken $x$ has a well-defined peak. As it can be seen in Fig.1 (bottom panel) the $x u_{V}(x)$ distribution peaks around $x \approx 0.2$, at the same value for which according to the left panel of the figure the SU(6) symmetry approximately holds. 

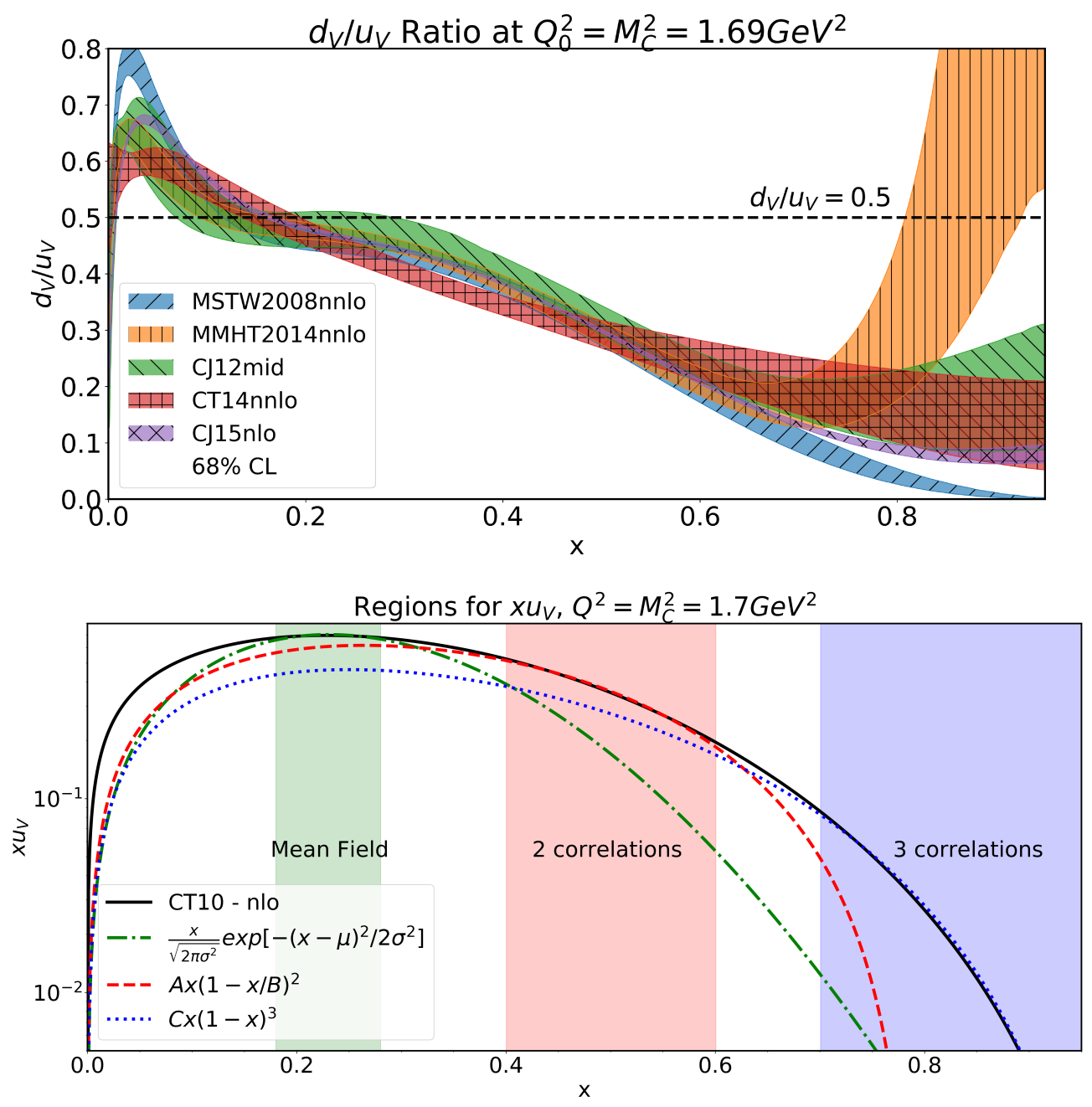

Figure 1: (top panel) The ratio of the down valence and up valence PDF for various PDF sets. (bottom panel) The graph shows the comparison of the $\mathrm{x}$ weighted valence $\mathrm{u}$-quark distribution fitted by three parametric functions corresponding to the mean field, $2 q-$ and $3 q$ - correlations.

In general, the peaking of the momentum distribution for a bound Fermi system is related to the total volume of the bound system, thus it characterizes the bulk of the interaction rather than mutual interaction among a limited number of constituents. This implies the importance of mean-field dynamics for the individual valence fermions in the domain where the peak is observed. The above phenomenology justifies our main assumption of the model according to which valence quark distribution in the nucleon is generated through three main mechanisms: mean field dynamics which is responsible for the peaking property of $x q_{V}(x)$ distribution and two- and three- quark short range correlations which are responsible for the generation of high $x$ distribution of valence quarks.

It is interesting that using parametric dependencies of these three mechanisms we can successfully reproduce the shape of the $x q_{V}(x)$ distribution for the up quarks as it is shown in Fig.1, (bottom panel). Furthermore, our next assumption is that the short-range quark correlations are generated due to hard gluon exchanges. Because of the vector nature of the exchange one obtains 
the selection rule according to which $q q$ correlations with opposite helicities will dominate in the partonic distribution. This selection rule is in some ways analogous to what was observed in nuclear physics when NN short range correlations were dominated by the proton-neutron component due to the tensor nature of the interaction[4]. In our model this helicity selection rule is responsible for the violation of SU(6) symmetry and in this way we predict that apparent di-quark symmetry of the nuclear structure is an emergent phenomenon of high $\mathrm{x}$ dynamics.

According to the considered model the three main diagrams of Fig.2 will contribute in the deep inelastic structure function of the nucleon.

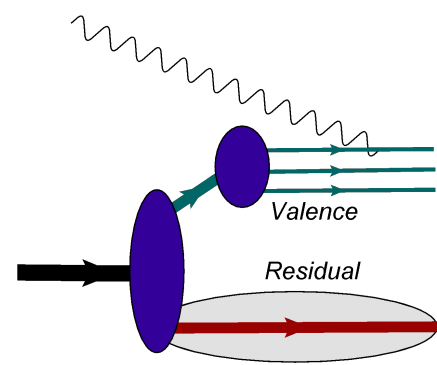

(a)

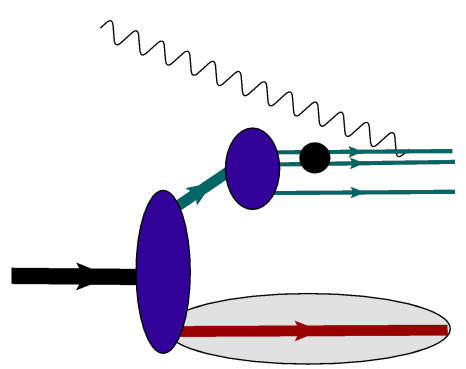

(b)

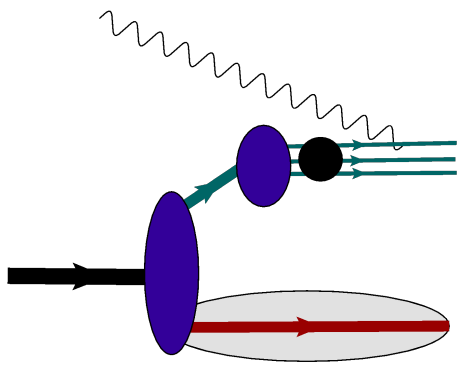

(c)

Figure 2: Mean field (a), two-valence quark (b) and three -valence quark correlation contribution to the deep-inelastic scattering off the nucleon, in the partonic picture.

As it follows from the figure the model assumes a certain universality of the residual structure, $R$, entering in all three mechanisms of generation of valence quark distribution Fig.2. This universality is reflected in the fact that one can fix its main properties within one of the approaches (say mean field model) and apply it in the description of $2 q$ and $3 q$ short range correlations. In principle it is possible to extract this distribution experimentally with dedicated measurements of tagged structure functions in semi-inclusive DIS processes.

\section{Valence Quark Distribution in Mean Field Approximation}

We derive the valence quark PDF by calculating nucleon structure function in DIS according to diagram of Fig.2(a). Here introducing the effective transition vertices and applying the Feynman diagrammatic rules one obtains for the scattering amplitude $A^{\mu}$ :

$$
A^{\mu}=\sum_{h_{V}, h_{1}} \frac{\bar{u}\left(k_{1}^{\prime}, h_{1}^{\prime}\right)\left(i e_{1} \gamma^{\mu}\right) u\left(k_{1}, h_{1}\right)}{k_{1}^{2}-m_{1}^{2}} \frac{\prod_{i=1}^{3} \bar{u}\left(k_{i}, h_{i}\right) \Gamma^{V \rightarrow 3 q} \chi_{V} \bar{\chi}_{V} \bar{\chi}_{R} \Gamma^{B \rightarrow V R} u\left(P, h_{N}\right)}{k_{V}^{2}-m_{V}^{2}} .
$$

Because of high energy kinematics of the scattering it is natural to project the covariant diagram on the light front considering positive light-cone energy propagation of intermediate states. This allows us to introduce the following light-front wave functions for nucleon transition into valence quark, $V$ and residual $R$ system, $\psi_{V R}$ as well as wave function of three valence quarks, $\psi_{3 q}$ as follows:

$$
\psi_{V R}\left(x_{V}, \mathbf{k}_{R, \perp}, x_{R}, \mathbf{k}_{V, \perp}\right)=\frac{\overline{\chi_{V}} \bar{\chi}_{R} \Gamma^{B \rightarrow V R} u\left(P, h_{N}\right)}{M^{2}-\frac{k_{V, \perp}^{2}+m_{V}^{2}}{x_{V}}-\frac{k_{R, \perp}^{2}+m_{R}^{2}}{x_{R}}}, \quad \psi_{3 q}\left(\left\{\beta_{i}, \mathbf{k}_{i, \perp}, h_{i}\right\}\right)=\frac{\prod_{i=1}^{3} \bar{u}\left(k_{i}, h_{i}\right) \Gamma^{V \rightarrow 3 q} \chi_{V}}{m_{V}^{2}-\sum_{i=1}^{3} \frac{k_{i, \perp}^{2}+m_{i}^{2}}{\beta_{i}}} .
$$


With the light-cone wave functions the scattering amplitude reduces to:

$$
A^{\mu}=\sum_{h_{1}, h_{V}} \bar{u}\left(k_{1}, h_{1}\right)\left(i e_{1} \gamma^{\mu}\right) u\left(k_{1}, h_{1}\right) \frac{\psi_{V R}\left(x_{V}, \mathbf{k}_{R, \perp}, x_{R}, \mathbf{k}_{V, \perp}\right)}{x_{V}} \frac{\psi_{3 q}\left(\left\{\beta_{i}, \mathbf{k}_{i, \perp}, h_{i}\right\}_{i=1}^{3}\right)}{\beta_{1}}
$$

Using the $A^{+}$component of the amplitude can calculate the nucleon structure function through:

$$
F_{2 N}\left(x, Q^{2}\right)=\frac{P \cdot q}{4 \pi\left(P^{+}\right)^{2}} \sum_{\left\{h_{i}, \tau_{i}\right\}} \int\left|A^{+}\right|^{2} \delta\left(k_{R}^{2}-m_{R}^{2}\right) \frac{d^{4} k_{R}}{(2 \pi)^{4}} \prod_{i=1}^{3} \delta\left(k_{i}^{2}-m_{i}^{2}\right) \frac{d^{4} k_{i}}{(2 \pi)^{4}} \delta^{(4)}\left(P+q-\sum_{i=1}^{3} k_{i}-k_{R}\right)
$$

and through the relation: $F_{2 N}(x)=\sum_{q} e_{q}^{2} x_{B} f_{q}\left(x_{B}\right)$ one obtains the valence quark distribution function:

$$
f_{q}\left(x_{B}\right)=\sum_{h_{i}} \int[d x]\left[d^{2} \mathbf{k}_{\perp}\right] e_{q}^{2} \boldsymbol{\delta}\left(x_{1}-x_{B}\right)\left|\psi_{3 q}\left(\left\{\beta_{i}, \mathbf{k}_{i, \perp}, h_{i}\right\}_{i=1}^{3}\right)\right|^{2}\left|\psi_{V}\left(x_{V}, \mathbf{k}_{R, \perp}, x_{R}, \mathbf{k}_{V, \perp}\right)\right|^{2} .
$$

To proceed with numerical evaluation of the PDFs we need a model for the $\psi_{3 q}$ and $\psi_{V}$ wave functions. The $3 \mathrm{q}$ system is modeled as relativistic coupled three harmonic oscillators, while the residual system, which in our case carries most of the nucleon mass and is non-relativistic, is described by a Gaussian wave function.

Once the analytic form of the wave functions are identified we fix the parameters of the wave function by comparing it with the $x q_{V}(x)$ distribution of the down quark. With the down quark PDF calculated the up quark PDF is obtained using the $S U(6)$ symmetry (i.e. $u_{V}\left(x, Q^{2}\right)=2 d_{V}\left(x, Q^{2}\right)$ ). Even though the extracted $d_{V}$ has greater uncertainties, we interpret the decrease of $\frac{d}{u}$ with $x$ due to the larger contribution of quark correlations in $u_{V}$ as compared to $d_{V}$. To estimate the maximal contribution from the mean-field we assume that all the height of the $x d_{V}(x)$ comes from the mean field dynamics and use Eq.(3.5) to fit both the height and position of the peak (Fig.3(left panel)), then evaluate $u_{V}\left(x, Q^{2}\right)=2 d_{V}\left(x, Q^{2}\right)$ (Fig.3(right panel)).
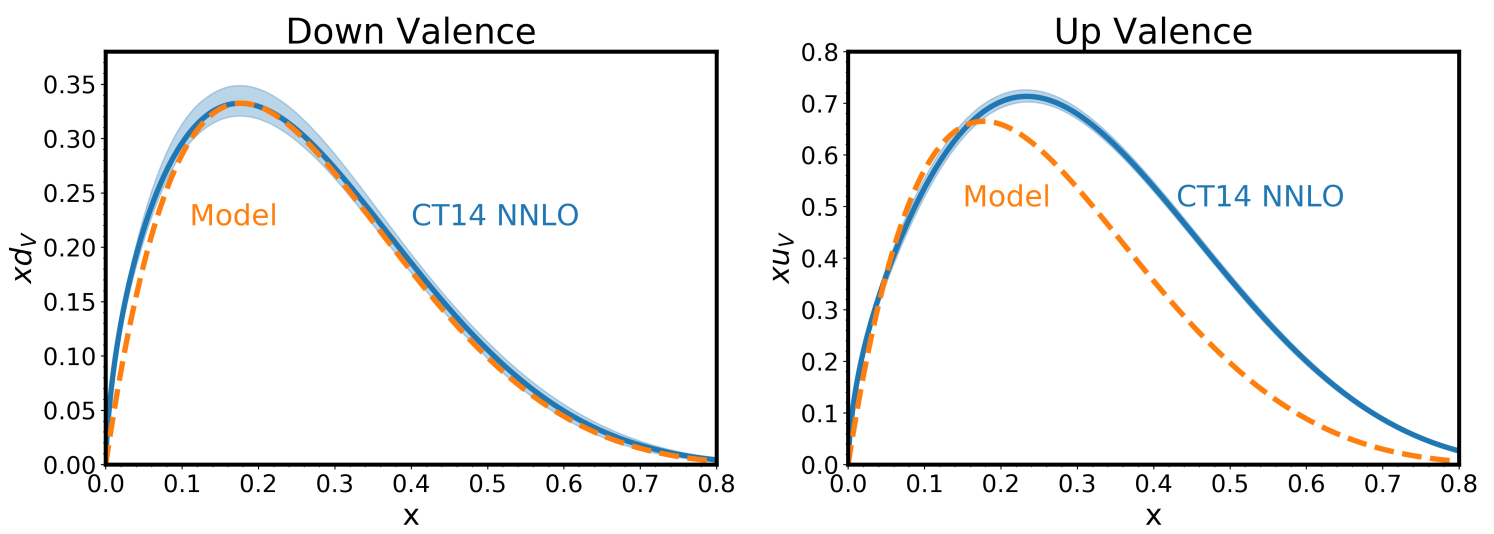

Figure 3: Comparison of the structure functions $x d_{V}$ and $x u_{V}$ (assuming $S U(6)$ ) from the model with CT14nnlo at $Q^{2}=M_{C}^{2} \simeq 1.7 \mathrm{GeV}^{2}$.

With this way we also estimate the minimal contribution from quark correlations since it is assumed that correlations do not contribute to $d_{V}(x)$ at $x \sim 0.2$. After evaluating the parameters of 
the wave function by this fitting procedure, we calculate the factors that the mean-field contributes into the values of $\int_{0}^{1} q_{V}(x) d x$ and $\int_{0}^{1} x q_{V}(x) d x$. For $d_{V}$ one obtains 0.83 and 0.126 compared to the actual values of 1 and 0.132 respectively. While for $u_{V}$ one evaluates 1.65 and 0.252 compared to the 2 and 0.323 , respectively. These numbers indicate that mean field captures $83 \%$ of normalization for both $d$ and $u$ quarks leaving $13 \%$ for quark correlations and $95.4 \%$ and $78 \%$ normalizations for the momentum carried by the valence quarks in the nucleon by $d$ and $u$ quarks, respectively. It is worth mentioning that $13 \%$ should be considered as the minimal contribution from quark correlations due to specific assumptions made for $d_{V}$.

\section{Acknowledgments}

We are thankful to Frank Vera for discussions. This work is supported by the US Department of Energy grant DE-FG02-01ER41172.

\section{References}

[1] S. J. Brodsky, T. Huang and G. P. Lepage, Hadronic wave functions and high momentum transfer interactions in quantum chromodynamics, Conf. Proc. C810816 (1981) 143.

[2] R. J. Holt and C. D. Roberts, Distribution Functions of the Nucleon and Pion in the Valence Region, Rev. Mod. Phys. 82 (2010) 2991 [1002.4666].

[3] L. Frankfurt, M. Sargsian and M. Strikman, Recent observation of short range nucleon correlations in nuclei and their implications for the structure of nuclei and neutron stars, Int. J. Mod. Phys. A23 (2008) 2991 [0806.4412].

[4] M. M. Sargsian, New properties of the high-momentum distribution of nucleons in asymmetric nuclei, Phys. Rev. C89 (2014) 034305 [1210 . 3280]. 